arithmetical skills. However, they neglect the neo-Fregean alternative axiomatization of arithmetic, based on Hume's principle. Frege arithmetic is arguably a more plausible start for a top-down approach in the psychological study of mathematical cognition than Peano arithmetic.

In the early days of developmental psychology, psychologists were in close contact with logicians and mathematicians working on the foundations of mathematics. Karl and Charlotte Bühler participated in the discussions of the Vienna Circle, which included among its members, Rudolf Carnap, Kurt Gödel, and Karl Menger. Piaget's work was heavily influenced by Bourbaki, the French structuralist school, and Piaget had many discussions with Jean Dieudonné, one of its leading members (see Piaget 1968; cf. Aczel 2007). In recent decades, developmental psychology and foundational work in mathematics have grown apart, sometimes resulting in outright antagonism (Dehaene 1997). Rips et al. restore the connection between two research fields. In their top-down approach, the cognitive math principles are based on the Dedekind-Peano axioms. They argue that these axioms or mathematically equivalent minor variations (e.g., the Least Number Principle) should be studied more seriously by psychologists. My major worry is that they have missed an important new development in foundational studies in mathematics.

Rips et al. briefly consider Frege's conception of numbers as sets of all equinumerous sets of objects, and rightly conclude that this view is untenable (Frege 1884/1974; 1893/1967). Frege's system was flawed, because of the inconsistency of the notorious Law V. However, Crispin Wright (1983) pointed out that Frege's arithmetic can be derived from Hume's principle (HP). Frege (1893/1967) contains all the essential inferences for a valid deduction of the laws of arithmetic, and Frege's inconsistent Law V can thus be sidestepped. The second-order Peano postulates can be derived in a consistent second-order system with only one extra-logical predicate N ("is the number of") and one non-logical axiom HP, stating that the number of Fs is equal to the number of Gs, in case there is a one-to-one correspondence between the Fs and the Gs. This result is known as Frege's theorem, and it implies that HP can serve as the basis of arithmetic (Heck 1993; Zalta 2008).

From a mathematical point of view, second-order Peano arithmetic (PA) and Frege arithmetic (FA) (second-order logic + HP) are almost equivalent (for their relative strength, see, e.g., Heck 2000). From an epistemological or cognitive point of view, PA and FA are very different. The basic concept in PA is the successor relation. PA is thus strongly related to the ordinal conception of number or to the cognitive abilities of enumeration and counting. FA, on the other hand, is based on equinumerosity, or oneto-one correspondence. The basic cognitive ability underlying HP is the ability to judge whether the objects of two sets can be put into a one-to-one relation, or, the ability to relate every object of a set to a single object of another set. For example, a set of knives is equinumerous to a set of forks if one can form pairs of forks and knives, without remaining forks or knives. Equinumerosity judgments are thus possible without enumerating the two collections. In the philosophy of mathematics, this discovery has triggered a lively epistemological debate on the question whether HP is an a priori or conceptual truth (Boolos 1997; Demopoulos 1998; Heck 2000). Neo-Fregeans claim that HP is central in mathematical knowledge.

The problem with the neo-Fregean program is that its claims about mathematical knowledge are not based on psychological evidence. Nevertheless, HP and Frege's theorem may be quite important in the empirical study of mathematical cognition. Since the 1970s, psychologists have regarded counting as the basis of numerical skills. Gelman and colleagues have argued that three principles underlie the ability to count: the one-toone correspondence principle, the stable-order principle, and the cardinality principle (Gelman \& Gallistel 1978; Gelman \& Greeno 1989; Gelman \& Meck 1983; Gelman et al. 1986).
These principles constitute an enumeration procedure and suffice to explain various numerical skills. However, the fact that these studies are largely based on the numerical skills of infants that have been taught to count at a very early age may have biased this research. It is perfectly possible that the ability to make one-to-one correspondence judgments (HP) is more basic and relevant than has generally been assumed. Two (arbitrarily chosen) examples should suffice to illustrate that HP is often overlooked as an explanation for certain numerical abilities.

Gordon (2004) carried out several matching tasks during his stay with the Pirahã. Gordon would put a certain number of objects $(1-10)$ below a line, and the participant had to put an equal amount of objects on the other side of the line. In general, there is a considerable decrease in performance for larger numbers, with one striking exception, namely, the line match. This would fit well with the participants having mastered a one-to-one correspondence procedure. As Gordon tampered with the matching condition, performance decreased. Gordon's results clearly indicate that non-numerates can employ a oneto-one correspondence procedure, without using enumeration (despite his claim to the contrary; Gordon 2004, p. 497). The uneven line match is especially noteworthy. If rectangles below the line were put in a line with unequal distances, performance was very good until four, and then dropped below $50 \%$ for five and six, and later went up again to almost correct performance for higher numbers. An explanation might be that for small numbers, the Pirahã use the more or less precise subitizing, with the effect that performance decreases rapidly above three. For larger sets, a one-to-one correspondence procedure takes over, leading to almost perfect performance. This is a hypothesis that can rather easily be tested, because one would assume that participants can be taught to use this strategy also for smaller numbers, with a resulting overall excellent performance.

Second, Jordan and Brannon (2006) have demonstrated that 7month-old infants can already recognize cross-modal one-to-one correspondences for low numerosities. Although Brannon and Jordan explain this result as evidence for a number representational system, it can more easily be interpreted as the mastery of $\mathrm{HP}$.

In conclusion, the claim made by Rips et al. that math schemas, roughly based on the Dedekind-Peano axioms, may be important in the study of (mature) mathematical cognition deserves further empirical scrutiny. However, there is a nontrivial, almost equivalent axiomatic approach that is arguably very different from a cognitive point of view. In a top-down approach towards mathematical cognition focusing on knowledge of mathematical principles, it seems more promising to start with a psychological study of one-to-one correspondence (FA or Heck's roughly equivalent system; Heck 2000), than with Peano's mathematical induction or commutativity.

\section{Bridging the gap between intuitive and formal number concepts: An epidemiological perspective}

\author{
doi:10.1017/S0140525X08005657
}

\section{Helen De Cruz}

Centre for Logic and Philosophy of Science, Free University of Brussels, 1050 Brussels, Belgium; and Centre for Logic and Analytical Philosophy, University of Leuven, 3000 Leuven, Belgium.

Helen.DeCruz@hiw.kuleuven.be

http://www.vub.ac.be/CLWF/members/helen/index.shtml

Abstract: The failure of current bootstrapping accounts to explain the emergence of the concept of natural numbers does not entail that no link exists between intuitive and formal number concepts. The epidemiology of representations allows us to explain similarities between intuitive and 
formal number concepts without requiring that the latter are directly constructed from the former.

Rips et al. have rightly pointed out a lack of fit between the properties of the natural numbers as defined by axiomatic systems in number theory and the unlearned representations of magnitude in infants. However, to conclude from this that there is "no dependency whatsoever" (sect. 6, para. 2) between them seems premature. Typically, the domains of intuitive knowledge that developmental psychologists have uncovered (such as intuitive psychology or number) are underdetermined. For example, although children are born with cognitive biases that lead them to attend to the actions and goals of others, it remains as yet unclear how they reliably develop a fully-fledged belief-desire psychology that differentiates an agent's mental states from the actual state of the world.

As an alternative to Rips et al.'s and to traditional bootstrapping approaches, it may be fruitful to examine number from the perspective of the epidemiology of representations. Its basic idea is that there is a strong causal link between the mental representations of the individual members of a culture and the public representations they share - in this case natural number concepts and their symbolic denotations (number words, numerical notation systems). To acquire a novel concept, learners partly draw on pre-existing knowledge. Thus, each time a cultural representation is transmitted, it has to pass the bottleneck of the pool of mental representations within the minds of individual learners. Representations with a poor fit to the pool of knowledge are less likely to be understood, and hence transmitted, than those with a good fit. Cognitive biases that are universal in humans likely play an important role in this process. As Nichols (2002) has demonstrated for etiquette norms, our universal feel of disgust for bodily excretions makes rules that limit contact with them (e.g., prohibitions to spit in public) more attractive than norms that do not stir our evolved emotional responses (e.g., placement of the napkin to the left or right of the plate). Importantly, Nichols (2002) does not claim that etiquette norms are directly based on or constructed from universal human predispositions. Rather, their good fit with our evolved drive to avoid disgusting situations has promoted their cultural success.

In the case of number, unlearned quantificational skills might similarly constrain and guide the cultural transmission of numerical concepts. If number concepts were based on axiom-like schemas, as Rips et al. suggest, we would expect some cultures to develop nonstandard numbers - which satisfy Peano's axioms in all respects but which we would yet not call numbers; however, apart from Western mathematics, there is no evidence that nonstandard models of arithmetic were ever developed. Unlearned intuitions of number may promote the cultural fitness of some numerical representations in favor of others. Evidence from educational psychology (Vlassis 2004) suggests that adolescents have difficulties grasping the concept of negative integers: they make more mistakes when solving equations that involve negative terms, and especially those that yield negative solutions. Although adults can compare the magnitudes of pairs of natural numbers quasi-automatically, their performance drops markedly when one or both digits are negative (Fischer 2003). These difficulties are hard to explain from a purely formal point of view, as the negative integers' properties are in many respects similar to the natural numbers', such as closure (i.e., $a+b$ is a natural number/integer for any natural number/integer $a$ and $b$ ), commutativity, and associativity. From an ecological point of view, however, conceptualizing negative integers is less relevant for organisms than conceptualizing positive quantities. If our evolved intuitions of number continue to play a role in learning processes, it becomes easier to understand why negative integers were historically less widespread than positive integers. Indeed, negative numbers were actively resisted despite their usefulness in calculations in cultures as disparate as 16th-century Europe, Han-dynasty China, and the medieval Islamic world.

What, then, is the relationship between our innate magnitude representations and natural numbers? One possibility which seems consistent with anthropological data is that although natural numbers are supported by unlearned inductive inferences (De Cruz 2006), there is considerable cultural variation in the degree to which public representations of number actually support them. For example, humans are equipped with the ability to discriminate between continuous and discrete quantities (Castelli et al. 2006). In some cultures (e.g., Western culture), children are confronted with a variety of symbolical representations for number, such as number words, Arabic digits, or even finger counting. These public representations provide external instantiations of the discreteness of natural numbers, leading children to understand that large numbers that are close together are yet distinct. Indeed, Western 5-year-olds who typically only count to 20 infer that numbers above their counting range apply to specific, unique cardinal values: if a set has 61 members, it cannot contain 65 elements (Lipton \& Spelke 2006). In contrast, in some Amazonian or Australian aboriginal cultures this distinction is not made, leading people growing up in these communities to rely on approximate numerical skills only. Consequently, they cannot discriminate between quantities if the ratio between them is small. While our intuitive quantification skills are not sufficient for natural number concept formation, they do support inductive inferences that promote an understanding of natural numbers in cultures that use symbolic representations that denote exact cardinalities.

\section{Not all basic number representations are analog: Place coding as a precursor of the natural number system}

\author{
doi:10.1017/S0140525X08005669
}

\section{Wim Fias and Tom Verguts \\ Department of Experimental Psychology, Ghent University, B-9000 Ghent, Belgium. \\ wim.fias@ugent.be http://expsy.ugent.be \\ tom.verguts@ugent.be http://users.ugent.be/ tverguts/}

Abstract: Rips et al.'s arguments for rejecting basic number representations as a precursor of the natural number system are exclusively based on analog number coding. We argue that these arguments do not apply to place coding, a type of basic number representation that is not considered by Rips et al.

We commend Rips et al.'s initiative to put to the fore how a conceptual understanding of the natural numbers is achieved by our cognitive system. This is a necessary step towards the integration of scientific progress in the now largely separate domains of basic number representations and more complex forms of numerical cognition. In this respect, it is a crucial question whether basic number representations constitute the basis for the development of a complete knowledge of the natural number system.

We do not agree, however, with the authors' conclusion that magnitude representations cannot be the precursor of understanding the properties of the natural number system. Our main point is that the authors are selective in regarding the magnitude representations they envisage. The authors' argument is exclusively built on analog magnitude representations. Although the biological reality of analog coding has been demonstrated (Roitman et al. 2007), there are reasons to believe that its functional importance in numerical cognition is limited. In a behavioral priming experiment, we have shown that in a naming task, dot displays evoke a priming pattern that is consistent with an analog magnitude code, but that Arabic digit primes 